\title{
RGS9-2 Modulates Responses to Oxycodone in Pain-Free and Chronic Pain States
}

\author{
Sevasti Gaspari',2, Valeria Cogliani', Lefteris Manouras², Ethan M Anderson', Vasiliki Mitsi', \\ Kleopatra Avrampou', Fiona B Carr' and Venetia Zachariou*,I \\ I'cahn School of Medicine at Mount Sinai, Fishberg Department of Neuroscience and Friedman Brain Institute, New York, NY, USA; ${ }^{2}$ University of \\ Crete Faculty of Medicine, Department of Basic Sciences, Heraklion, Greece
}

\begin{abstract}
Regulator of G-protein signaling 9-2 (RGS9-2) is a striatal-enriched signal-transduction modulator known to have a critical role in the development of addiction-related behaviors following exposure to psychostimulants or opioids. RGS9-2 controls the function of several G-protein-coupled receptors, including dopamine receptor and mu opioid receptor (MOR). We previously showed that RGS9-2 complexes negatively control morphine analgesia, and promote the development of morphine tolerance. In contrast, RGS9-2 positively modulates the actions of other opioid analgesics, such as fentanyl and methadone. Here we investigate the role of RGS9-2 in regulating responses to oxycodone, an MOR agonist prescribed for the treatment of severe pain conditions that has addictive properties. Using mice lacking the Rgs9 gene (RGS9KO), we demonstrate that RGS9-2 positively regulates the rewarding effects of oxycodone in pain-free states, and in a model of neuropathic pain. Furthermore, although RGS9-2 does not affect the analgesic efficacy of oxycodone or the expression of physical withdrawal, it opposes the development of oxycodone tolerance, in both acute pain and chronic neuropathic pain models. Taken together, these data provide new information on the signal-transduction mechanisms that modulate the rewarding and analgesic actions of oxycodone.

Neuropsychopharmacology (2017) 42, I548-1556; doi:I0.1038/npp.2017.4; published online 8 February 2017
\end{abstract}

\section{INTRODUCTION}

Synthetic and semisynthetic opioids, including fentanyl, hydromorphine, and oxycodone are prescribed for the treatment of severe acute or chronic pain conditions (Carise et al, 2007; Ling et al, 2011; Hermanns et al, 2012). Oxycodone in particular (available in several formulations, alone or in combination with non-narcotic analgesics) has been used as an alternative to morphine for the management of severe pain. The increasing numbers of oxycodonedependent patients, and the rising incidents of deaths from oxycodone overdose (Carise et al, 2007; Comer et al, 2007; Spiller et al, 2009; Ling et al, 2011; Butler et al, 2011; Dart et al, 2015; Ray et al, 2016) highlight the need for a better understanding of the mechanism of action of oxycodone analgesia and the factors that determine vulnerability to oxycodone dependence and addiction.

Whereas there is a lot of information from in vitro work on the signaling properties of a range of mu opioid receptor (MOR)-targeting compounds, in vivo research on MOR agonist signal-transduction mechanisms has focused largely

* Correspondence: Dr V Zachariou, Icahn School of Medicine at Mount Sinai, Fishberg Department of Neuroscience and Friedman Brain Institute, One Gustave L. Levy Place, Box 1065, New York, NY I0029, USA, Tel: 212659 86I2, Fax: 2126598574 ,

E-mail: venetia.zachariou@mssm.edu

Received 8 August 2016; revised 28 November 2016; accepted 23 December 2016; accepted article preview online II January 2017 on the study of morphine or heroin (Bailey and Connor, 2005; Muller and Unterwald, 2004; Koch et al, 2005; Raehal and Bohn, 2005; Pradhan et al, 2006; Walwyn et al, 2010; Quillinan et al, 2011). Recently, several preclinical studies demonstrated the potent effect of oxycodone exposure in the development of addiction-related behaviors and adaptations in synaptic plasticity, in adolescent and adult animals (Niikura et al, 2013; Zhang et al, 2013, 2015). These studies have demonstrated that oxycodone has prominent effects on the expression of genes modulating synaptic plasticity in several brain regions, including the dorsal striatum and the hippocampus. Importantly, oxycodone self-administration in early life affects the sensitivity to the rewarding and analgesic actions of the drug in the adult life (Zhang et al, 2016). The preclinical and epidemiological findings on oxycodone abuse highlight the need to study the neurochemical and molecular actions of this drug in pain-free as well as in chronic pain states.

RGS9-2 is enriched in the striatum, and has a potent role in the modulation of functional responses of several G-protein-coupled receptors (GPCRs), including MOR and dopamine receptors (Zachariou et al, 2003; Rahman et al, 2003; Kovoor et al, 2005; Terzi et al, 2009; Kimple et al, 2011). RGS9-2 binds to activated $\mathrm{G} \alpha$ subunits and controls the duration of signal transduction, by regulating how long $\mathrm{G} \alpha$ and $\beta \gamma$ subunits are available to their effectors. In addition, the binding of RGS9-2 to $\mathrm{G} \alpha$ subunits may prevent activation of their effectors, without affecting $\beta \gamma$ complex's 
actions (Hollinger and Hepler, 2002; Traynor et al, 2009; Kimple et al, 2011). We previously showed that striatal RGS9-2 controls MOR signal transduction and desensitization (Zachariou et al, 2003; Psifogeorgou et al, 2007; Psifogeorgou et al, 2011; Gaspari et al, 2014). Several biochemical and in vivo studies suggest that RGS9-2 forms complexes with $\mathrm{G} \alpha$ subunits, the $\mathrm{G} \beta 5$ protein and other adaptor or scaffolding molecules to dynamically modulate the acute and chronic actions of the opioid analgesic morphine (Psifogeorgou et al, 2007; Anderson et al, 2007; Jayaraman et al, 2009; Psifogeorgou et al, 2011; Terzi et al, 2011; Gaspari et al, 2014). These RGS9-2 complexes affect several aspects of morphine addiction-like behavior in rodents, by negatively modulating reward and physical dependence, whereas they also affect analgesia and the development of analgesic tolerance (Zachariou et al, 2003; Terzi et al, 2009; Gaspari et al, 2014). Interestingly, RGS9-2 has a complex role in the regulation of MOR function, as suggested by more recent findings showing that RGS9-2 acts as a positive modulator of the analgesic effects of methadone and fentanyl (Psifogeorgou et al, 2011). Furthermore, recent studies reveal two SNPs located in the promoter region of Rgs9 gene significantly associated with heroin dependence, highlighting the translational aspect of the above-stated research (Zhu and Zhang, 2015).

Chronic pain leads to plasticity in several brain networks involved in reward, mood, and motivation (Baliki et al, 2012; Schwartz et al, 2014; Yalcin et al, 2014; Baliki and Apkarian, 2015; Mitsi and Zachariou, 2016). We therefore hypothesized that chronic pain states may influence sensitivity to the behavioral effects of oxycodone. Neuropathic pain is a severe chronic disorder, resulting from nerve damage due to injury or various central nervous system disorders. It is characterized by several symptoms, including dysesthesia, mechanical and cold allodynia, hyperalgesia, and often coexists with anxiety and depression (Gruccu, 2007; Shields et al, 2003; Stratinaki et al, 2013; Terzi et al, 2014; Mitsi and Zachariou, 2016).

Here we investigated the role of RGS9-2 in mediating the behavioral responses to oxycodone. Our findings suggest that RGS9-2 acts a positive modulator of oxycodone reward, in both pain-free and neuropathic pain states. Although oxycodone promotes a similar somatic withdrawal syndrome in mice to that observed with morphine (Zachariou et al, 2003), RGS9-2 does not affect the expression of somatic withdrawal syndrome to oxycodone. Finally, our study shows that RGS9-2 delays the development of oxycodone tolerance both under pain-free and neuropathic pain states. Overall, these findings provide new information on the cellular mechanisms modulating oxycodone actions and support the hypothesis that distinct modulatory mechanisms control behavioral responses to opiate analgesics.

\section{MATERIALS AND METHODS}

\section{Animals}

Two- to three-month-old male C57BL/6 mice (Jackson Labs) were used for biochemical studies. Behavioral studies were performed using adult (2-3-month-old) male RGS9-wildtype (RGS9WT) and RGS9-knockout (RGS9KO) mice derived from homozygote breeding from mice backcrossed
20 generations to $\mathrm{C} 57 \mathrm{BL} / 6$ background (Zachariou et al, 2003). Animals were housed in a $12 \mathrm{~h}$ dark-light cycle room according to the IACUC committee of Icahn School of Medicine. For all manually scored behavioral assays (hotplate test, oxycodone withdrawal, Von Frey testing) experimenters were blinded to the genotype.

\section{Locomotor Activity Assay}

Mice were habituated to the locomotor activity apparatus (Med Associates, VT) for $30 \mathrm{~min}$ each day for 3 consecutive days after receiving a subcutaneous saline injection. Following that, oxycodone was administered for 3 or 4 consecutive days, once per day, and ambulatory activity was monitored for $30 \mathrm{~min}$ immediately after drug injection as described earlier (Charlton et al, 2008).

\section{Conditioned Place Preference Test}

An unbiased place conditioning procedure was performed as described in earlier studies (Zachariou et al, 2003; Gaspari et al, 2014). Briefly, baseline preference was monitored for $20 \mathrm{~min}$, animals were conditioned to the drug paired side or to the saline paired side for $45 \mathrm{~min}$ on alternate days. After 6 conditioning days animals were tested again for $20 \mathrm{~min}$, having free access to both chambers. Preference was determined as time spent in the drug-paired compartment after conditioning minus the time spent in this compartment at baseline. Animals showing strong bias (over $250 \mathrm{~s}$ ) for one side at baseline were excluded from the study. For the oxycodone reinstatement studies (Figure 2b), mice were tested for baseline preference (day 1, for $20 \mathrm{~min}$ ) and then they were conditioned with saline in the morning ( $45 \mathrm{~min}$ ) and with a high dose of oxycodone $(5 \mathrm{mg} / \mathrm{kg}$, subcutaneously) in the afternoon ( $45 \mathrm{~min}$ ) for 2 consecutive days. Place preference was tested the day after the last conditioning session, for $20 \mathrm{~min}$. The extinction section involved saline injections paired to the saline side (morning, $45 \mathrm{~min}$ ) and the drug side (afternoon, $45 \mathrm{~min}$ ) for 5 days a week, for 4 weeks, starting $24 \mathrm{~h}$ after conditioned place preference (CPP) testing. Mice were evaluated for place preference once a week (20 min testing), until extinction was observed (week 4). For the reinstatement session, mice were injected with a low dose of oxycodone ( $1.5 \mathrm{mg} / \mathrm{kg}$, subcutaneously) and place preference was monitored for $20 \mathrm{~min}$.

\section{Hot-Plate Assay}

Analgesia was measured using a $52^{\circ} \mathrm{C}$ hot-plate apparatus (IITC Life Sciences, CA), as described previously (Gaspari et al, 2014). Animals were habituated in the room for $1 \mathrm{~h}$ and then tested for baseline latency to jump or paw lick. Oxycodone was then injected subcutaneously and $30 \mathrm{~min}$ later mice were placed on the hot-plate apparatus and latencies to lick the hind paw or jump were monitored. For tolerance studies this procedure was repeated for 6 consecutive days using a high oxycodone dose $(20 \mathrm{mg} / \mathrm{kg})$. All hot-plate data are expressed as \% of maximal possible effect $(\mathrm{MPE}=($ latency - baseline $) /($ cutoff - baseline $))$. A cutoff time of $40 \mathrm{~s}$ was used in all hot-plate experiments to avoid tissue damage and inflammation. 


\section{Opiate Withdrawal Paradigm}

For opiate withdrawal assays, mice were injected with increasing oxycodone doses every $12 \mathrm{~h}$ for 4 consecutive days (day 1: $20 \mathrm{mg} / \mathrm{kg}$; day 2: $40 \mathrm{mg} / \mathrm{kg}$; day 3: $60 \mathrm{mg} / \mathrm{kg}$; day 4: $80 \mathrm{mg} / \mathrm{kg}$ ). On day 5 mice were injected with $80 \mathrm{mg} / \mathrm{kg}$ oxycodone in the morning and withdrawal was precipitated $3 \mathrm{~h}$ later, using naloxone (NLX) hydrochloride (1 mg/kg, subcutaneously; Sigma, MO). Withdrawal signs (jumps, wet dog shakes, tremor, diarrhea, weight loss) were monitored for $30 \mathrm{~min}$, starting immediately after NLX administration. We monitored the number of jumps, wet dog shakes, and diarhhea events observed in the $30 \mathrm{~min}$ period, and \% change in weight before and $30 \mathrm{~min}$ post withdrawal for weight loss. For tremor and ptosis, we monitored the presence of a sign at the beginning of each $5 \mathrm{~min}$ interval during the monitoring period.

\section{Spared Nerve Injury Model}

The spared nerve injury (SNI) operation was performed under Avertine (2,2,2-tribromoethanol; Sigma-Aldrich) general anesthesia (Mitsi et al, 2015). With the help of a stereomicroscope, skin incision of the left hindlimb at midthigh level followed by muscle layers separation revealed the sciatic nerve and its three branches. The common peroneal and the sural nerves were carefully ligated with 6.0 silk suture (Ethicon; Johnson \& Johnson Intl.) transected and 1-2 mm sections of these nerves were removed, while the tibial nerve was left intact. Skin was then closed with silk 4.0 sutures (Ethicon; Johnson \& Johnson Intl).

\section{Von Frey Test for Mechanical Allodynia}

For the assessment of mechanical allodynia, we used Von Frey testing (Shields et al, 2003; Mitsi et al, 2015) with ascending forces expressed in grams (0.1-3.6 g; Electronic von Frey Anesthesiometer; IITC). Each filament was applied five times in a row against the lateral area of the paw. Hindpaw withdrawal or licking induced by the filament was defined as a positive allodynia response. A positive response in three out of five repetitive stimuli was defined as the allodynia threshold. Mice were habituated to the Von Frey apparatus for $30 \mathrm{~min}$ every day for 10 days, before the SNI surgeries. For the tolerance studies, mice were injected subcutaneously for 17 consecutive days with $3 \mathrm{mg} / \mathrm{kg}$ oxycodone and allodynia was assessed $1 \mathrm{~h}$ after the injection.

\section{Western Blot Analysis}

For western blot analysis, nucleus accumbens (NAc) and dorsal striatum (DS) punches were dissected with a 14 gauge syringe needle from 1-mm-thick coronal sections of mouse brain, as described before (Psifogeorgou et al, 2011; Gaspari et al, 2014). Samples were run in a $10 \%$ polyacrylamide gel, and then transferred to a nitrocellulose membrane (Bio-Rad $4.45 \mu \mathrm{m})$ for $1 \mathrm{~h}$. Membranes were incubated in blocking buffer (3\% filtered non-fat dry milk, in PBS) and incubated overnight at $4^{\circ} \mathrm{C}$ in $\mathrm{PBS}$ with primary antibody. The following antibodies were used: rabbit anti-RGS9-2 (Psifogeorgou et al, 2011), a rabbit anti-GAPDH (Cell Signaling; no. 5174), a rabbit anti- $\beta$-actin (Cell Signaling; no. 4967). $\beta$-Actin or GAPDH were used as loading controls. The next day membranes were incubated with goat antirabbit peroxidase-labeled secondary antibody (Jackson Laboratories; no. 111-035-003; $1: 10.000)$ in the blocking buffer. The bands were visualized using Super Signal West Pico Extended Duration Substrate (Pierce). Bands were quantified using the Image J Software. For all western blot analysis, sample optical density was normalized to loading control and then expressed as the percentage of the salinetreated group.

\section{Statistical Analysis}

For experiments monitoring behavior of the same group of mice over time (locomotor sensitization, CPP reinstatement, and tolerance experiments), we used two-way ANOVA repeated-measures followed by Bonferroni post hoc tests. For SNI-CPP experiments and acute oxycodone analgesia that involved plotting several doses (each dose was tested in a separate group of animals), we used two-way ANOVA followed by Bonferroni post hoc tests. For withdrawal experiments we used multiple $t$-test analysis. Finally, for western blot analysis studies, the CPP experiment testing $1 \mathrm{mg} / \mathrm{kg}$ of oxycodone (Figure 2a), and the morphine analgesia assays, we used $t$-test. Effects were considered significant at $p<0.05$, all data were expressed as mean \pm SEM. $F$ values and $p$-values for each experiment are described in the figure legends.

\section{RESULTS}

When administered repeatedly, drugs of abuse produce a progressive enhancement of locomotor response (locomotor sensitization). We used the RGS9KO line to investigate the behavioral consequences of Rgs9 deletion in an oxycodone locomotor sensitization paradigm. As shown in Figure 1a, $\mathrm{KO}$ of the Rgs9 gene does not affect basal locomotor activity nor the acute response to a high oxycodone dose $(3 \mathrm{mg} / \mathrm{kg}$ subcutaneously). However, RGS9KO mice are less sensitive than RGS9WT controls to the locomotor-sensitizing actions of oxycodone. We next used western blot analysis to determine if repeated oxycodone administration affects the expression of RGS9-2 in the NAc and DS. C57BL/6 mice were habituated to the locomotor chamber for 3 consecutive days, followed by 3 additional days of oxycodone treatment ( $2 \mathrm{mg} / \mathrm{kg}$, subcutaneously). On day 3, NAc and DS tissues were collected $2 \mathrm{~h}$ after drug administration (Figure $1 \mathrm{~b}$ ). Western blot analysis of these samples revealed that this treatment leads to the downregulation of RGS9-2 protein levels both in NAc and DS (Figures $1 \mathrm{c}$ and $\mathrm{d}$ ).

We next investigated the role of RGS9-2 in the rewarding actions of oxycodone. Our earlier work revealed a negative modulatory role of RGS9-2 in the rewarding actions of morphine (Zachariou et al, 2003; Gaspari et al, 2014). Here our data (Figure 2a) suggest that RGS9-2 acts as a positive modulator of oxycodone actions in the CPP paradigm, as RGS9KO mice are less sensitive to the rewarding actions of this drug ( $1 \mathrm{mg} / \mathrm{kg}$, subcutaneously). At higher doses both genotypes develop place preference to oxycodone (Figure $2 \mathrm{~b}$ ). We also assessed the role of RGS9-2 in the extinction and reinstatement of oxycodone place 


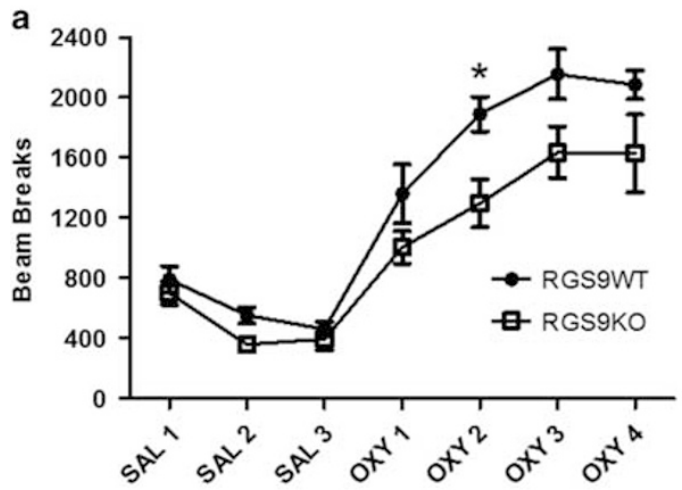

\section{b}
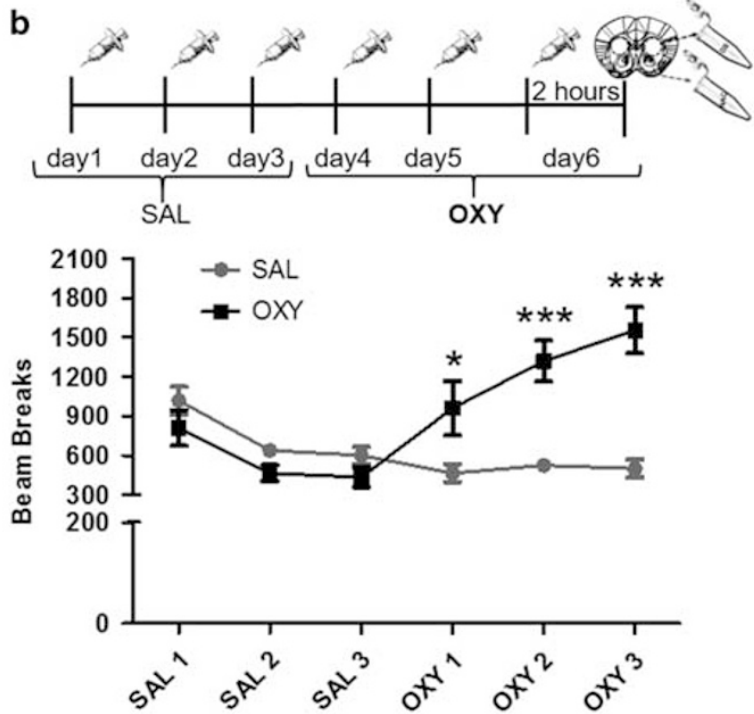

C SAL OXY SAL OXY

d RGS9-2 (76 kD)
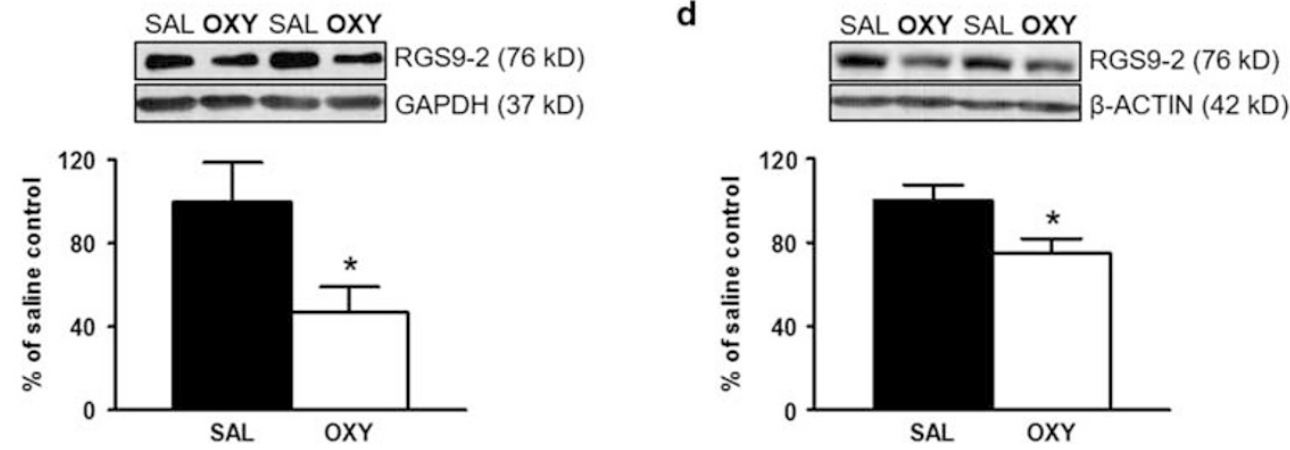

Figure I Regulator of G-protein signaling 9-2 (RGS9-2) modulates the locomotor-sensitizing actions of oxycodone. (a) RGS9-knockout (KO) mice are less sensitive to the locomotor-sensitizing actions of oxycodone compared with their RGS9-wild-type (WT) controls (dose: $3 \mathrm{mg} / \mathrm{kg}$, two-way analysis of variance (ANOVA) repeated-measures followed by Bonferroni posthoc test, $F(I, 48)=8.22$, $\left.{ }^{*} p<0.05\right)$. (b) The schematic on top shows the experimental design for tissue collection for our western blot analysis studies. The graph shows the locomotor responses of the cohort used for western blot analysis. Mice were habituated to the locomotor chamber for 3 consecutive days followed by 3 additional days of oxycodone administration (dose: 2 mg/kg, two-way ANOVA repeated-measures followed by Bonferroni posthoc test, $\mathrm{F}(\mathrm{I}, 40)=4.93$, $* p<0.05$, $* * * * p<0.00 \mathrm{I}$ ). On day 3 , nucleus accumbens (NAc) and dorsal striatum (DS) tissues were collected $2 \mathrm{~h}$ after drug administration. Western blot analysis reveals that RGS9-2 is downregulated in the NAc (c) and DS (d) of mice treated for 3 consecutive days with $2 \mathrm{mg} / \mathrm{kg}$ oxycodone (OXY) compared with saline (SAL)-treated controls $(t$-test, $t(19)=2.429$, * $p<0.05$ for NAc and $t(9)=2.433, * p<0.05$ for DS). GAPDH, glyceraldehyde 3-phosphate dehydrogenase.

preference. In this case we used a high dose of oxycodone $(5 \mathrm{mg} / \mathrm{kg})$, which induces CPP in both WT and mutant mice. Both genotypes extinguished oxycodone CPP after a month of saline training; however, only RGS9WT mice reinstated CPP following treatment with a low $(1.5 \mathrm{mg} / \mathrm{kg})$ oxycodone dose (Figure $2 \mathrm{~b}$ ). Thus, RGS9-2 has a positive modulatory role in the acquisition and reinstatement of oxycodone CPP.

We also investigated if RGS9-2 contributes to the rewarding actions of oxycodone under chronic pain conditions. We used the SNI model of neuropathic pain, and 2 weeks after the induction of nerve injury (when the animals have developed pain-like behaviors, but not depression-like behaviors; Terzi et al, 2014), we conditioned RGS9WT and RGS9KO mice to oxycodone. The neuropathic pain-like state decreased sensitivity to oxycodone place preference in RGS9WT mice, as they did not respond to the $1 \mathrm{mg} / \mathrm{kg}$ dose that conditions CPP in the pain-free state. RGS9KO mice did not respond to this dose either. When a higher dose was used, we found a similar phenotype to that observed under pain-free states: RGS9WT mice develop a significant place preference, while the RGS9KO show no significant preference to this dose of the drug (Figure 2c; $2 \mathrm{mg} / \mathrm{kg}$ : RGS9WT-initial preference of the drug side $=427 \pm 28$, conditioned preference $=647 \pm 30, t(10)=5.95, p<0.0001$; RGS9KO-initial preference $=490 \pm 35$, conditioned preference $=560 \pm 22, t(8)=1.96, p=0.085)$. At a higher dose (3 mg/kg) SNI groups of both RGS9WT and RGS9KO mice develop CPP to oxycodone (Figure 2c).

Our earlier work had also shown that KO of Rgs9 exacerbated several symptoms of somatic morphine withdrawal, suggesting that RGS9-2 opposes the development of physical dependence (Zachariou et al, 2003; Gaspari et al, 2014). To determine if RGS9-2 has a similar role in the development of oxycodone physical dependence, we monitored somatic symptoms for $30 \mathrm{~min}$ following NLX-precipitated withdrawal. Oxycodone was administered 
a Oxycodone CPP (1mg/kg)

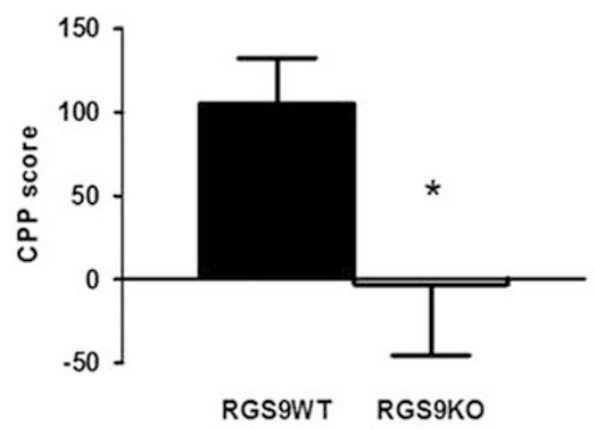

b
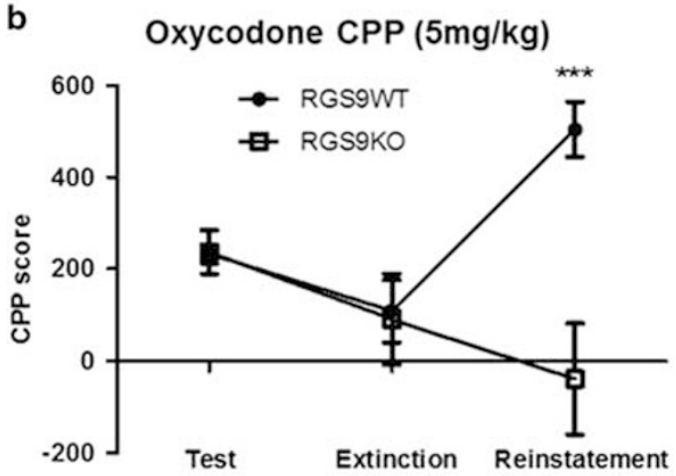

c SNI - Oxycodone CPP

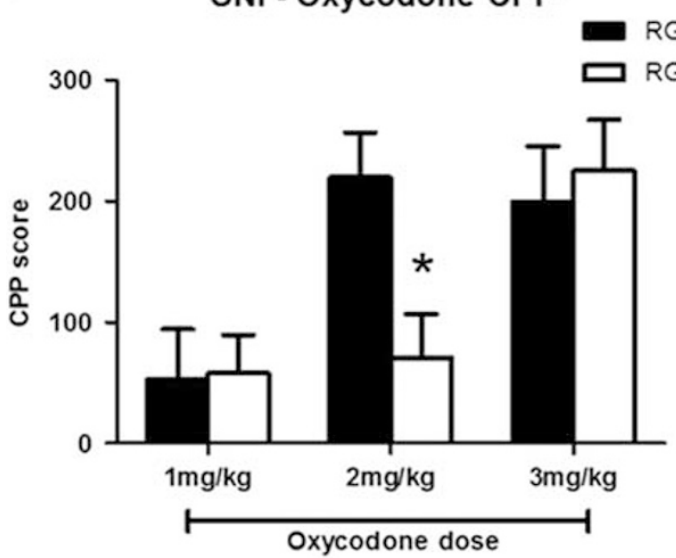

Figure 2 Regulator of G-protein signaling 9-2 (RGS9-2) modulates the rewarding actions of oxycodone. RGS9-2 acts as a positive modulator of oxycodone in the conditioned place preference (CPP) paradigm. (a) RGS9knockout $(\mathrm{KO})$ mice are less sensitive to the rewarding actions of oxycodone (dose: I mg/kg, t-test, $t(17)=2.249$, *p<0.05). (b) Furthermore, RGS9-2 has a positive modulatory role in the reinstatement of oxycodone CPP. Both genotypes developed CPP using a high dose of oxycodone $(5 \mathrm{mg} / \mathrm{kg})$ and extinguished preference after a month of saline training; however, only RGS9-wild-type (WT) mice reinstated CPP following a low $(1.5 \mathrm{mg} / \mathrm{kg}$ ) dose of oxycodone (two-way analysis of variance (ANOVA) repeated-measures followed by Bonferroni post hoc tests, F $(\mathrm{I}, \mathrm{I} 6)=5.5 \mathrm{I}$, ${ }^{* * * *} \mathrm{p}<0.00 \mathrm{I}$ ). (c) Using the spared nerve injury (SNI) model of neuropathic pain, we demonstrate that RGS9WT and RGS9KO do not develop place preference to a low dose of oxycodone (I mg/kg). At a higher dose $(2 \mathrm{mg} / \mathrm{kg})$, only RGS9WT mice develop preference, whereas both genotypes condition place preference at $3 \mathrm{mg} / \mathrm{kg}$ (two-way ANOVA followed by Bonferroni post hoc test, $F(I, I 6)=5.5 \mathrm{I}$, $* p<0.05)$.

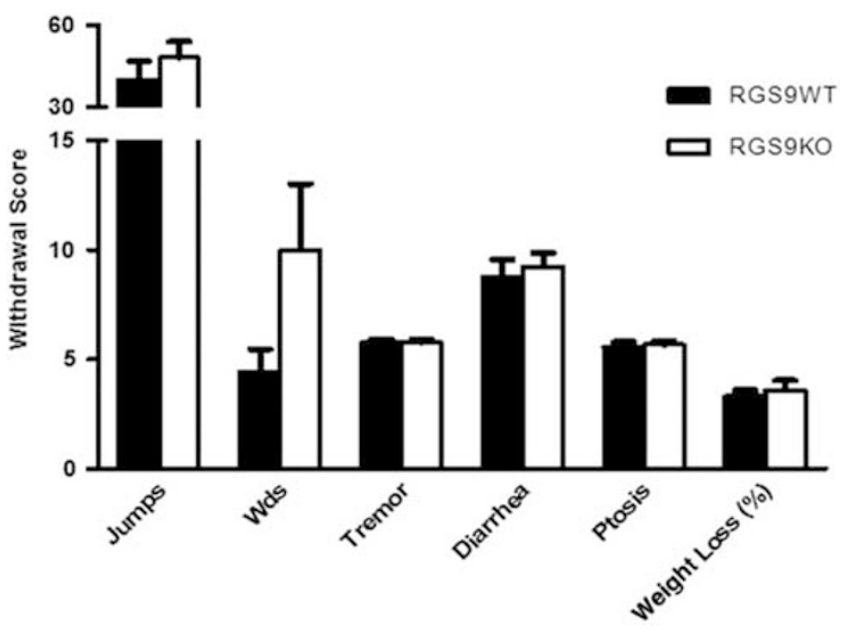

Figure 3 Knockout (KO) of the Rgs9 gene does not affect the intensity of oxycodone withdrawal symptoms. We used a paradigm of naloxone (NLX)-precipitated withdrawal to determine if regulator of G-protein signaling 9-2 (RGS9-2) has a role in oxycodone physical dependence. Oxycodone was administered for 5 days, twice a day, at increasing doses $(20,30,40,60$, and $80 \mathrm{mg} / \mathrm{kg})$. We monitored several somatic withdrawal symptoms (jumps, wet dog shakes, tremor, diarrhea, ptosis, and weight loss) for a $30 \mathrm{~min}$ period after $\mathrm{NLX}(\mathrm{I} \mathrm{mg} / \mathrm{kg})$ administration. Oxycodone produced a similar withdrawal behavior to the one observed with morphine, and onset and intensity of symptoms were comparable between RGS9KO mice and their RGS9-wild-type (WT) controls. RGS9KO mice showed a nonsignificant trend for a greater number of wet dog shakes (WDS) (multiple t-tests, Jumps: $t(16)=0.8833, p>0.05$, WDS: $t(16)=1.742$, $p>0.05$, Tremor: $t(16)=0, p>0.05$, Diarrhea: $t(16)=0.44 \mid 1, p>0.05$, Ptosis: $t(16)=0.3780, p>0.05$, weight loss $(\%): t(\mid 6)=0.5066, p>0.05)$.

for 5 days, twice a day, at increasing doses $(20,30,40,60$, and $80 \mathrm{mg} / \mathrm{kg}$ ). Although oxycodone produces similar withdrawal behavior to that seen for morphine, ablation of the $R g s 9$ gene did not affect the onset or intensity of withdrawal symptoms (Figure 3 ).

Finally, we investigated the role of RGS9-2 in the analgesic effects of oxycodone. We used the hot-plate assay to assess the consequences of global $\operatorname{Rgs} 9 \mathrm{KO}$ on the acute analgesic actions of oxycodone. Our previous work demonstrated that RGS9KO mice show increased responses to morphine in the hot-plate test (Zachariou et al, 2003). Here we found that KO of $R g s 9$ does not affect the acute analgesic effects of oxycodone in the $52^{\circ} \mathrm{C}$ hot-plate assay (Figure 4a). The top panel in Figure 4a shows that consistent with our earlier findings (Zachariou et al, 2003; Psifogeorgou et al, 2011), RGS9KO mice are more sensitive to the analgesic effect of morphine $(15 \mathrm{mg} / \mathrm{kg}$, subcutaneously). Western blot analysis showed that acute oxycodone treatment $(15 \mathrm{mg} / \mathrm{kg})$ has no effect on RGS9-2 expression in the NAc of C57Bl/6 mice (Figure $4 \mathrm{~b}$, NAc tissue dissected $2 \mathrm{~h}$ post oxycodone treatment). However, we found that RGS9-2 has a protective role towards the development of oxycodone tolerance, as RGS9KO mice become tolerant to the analgesic effects of the drug earlier than RGS9WT animals (Figure 5a). As shown on the top panel and consistent with our earlier findings, RGS92 has the opposite role in the modulation of morphine tolerance, as RGS9KO mice respond to repeated morphine treatment at 4 days, while their WT controls show analgesic tolerance. Furthermore, protein levels of RGS9-2 are 
a

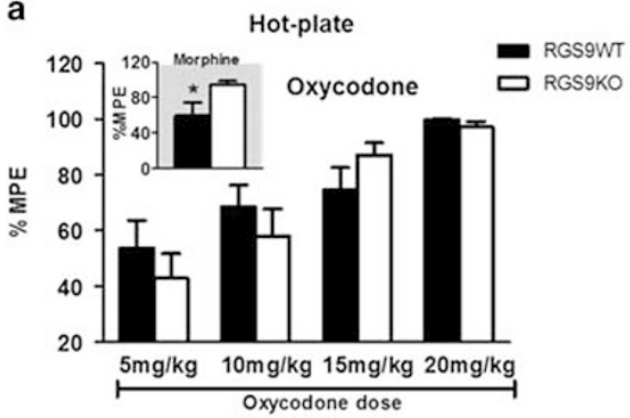

b

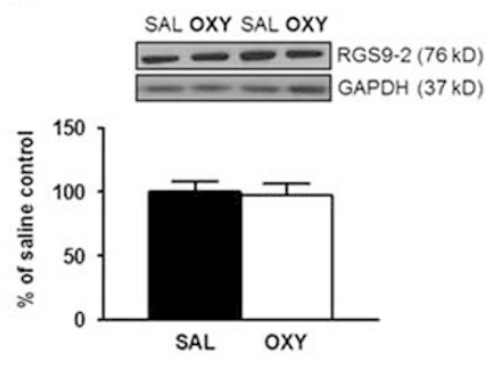

Figure 4 Regulator of G-protein signaling 9-2 (RGS9-2) does not affect the acute analgesic actions of oxycodone. (a) Ablation of the Rgs9 gene does not affect the acute analgesic effects of oxycodone in the hot-plate assay (each dose corresponds to a separate group of animals, two-way analysis of variance (ANOVA) followed by Bonferroni post hoc tests, $\mathrm{F}(\mathrm{I}, 74)=0.16, p>0.05)$. Upper panel: RGS9-knockout (KO) mice are more sensitive to the analgesic actions of morphine (dose: $15 \mathrm{mg} / \mathrm{kg}$, t-test, $t(7)=2$, *p<0.05). (b) Acute administration of a high dose of oxycodone $(15 \mathrm{mg} / \mathrm{kg}$ ) did not have any effect on RGS9-2 protein levels in the nucleus accumbens (NAc) (t-test, $t(19)=0.23, p>0.05)$. OXY, oxycodone; SAL, saline.

upregulated in the NAc of mice tolerant to oxycodone (Figure 5b).

The hot-plate test is a measure of acute analgesia, whereas oxycodone is often prescribed for chronic pain conditions. To determine if RGS9-2 affects the development of oxycodone tolerance under chronic pain states, we used the SNI paradigm and monitored responses to the antiallodynic effects of oxycodone in the Von Frey assay for mechanical allodynia. Our earlier work has shown that RGS9-2 affects Von Frey responses the first week after nerve injury, but by day 10, mechanical allodynia levels are not different from those observed in RGS9WT mice (Terzi et al, 2014). Here we started the treatment with oxycodone on day 15, when mechanical allodynia levels were identical between genotypes. The drug was administered once a day for 17 consecutive days, and Von Frey responses were monitored $1 \mathrm{~h}$ after drug injection. Consistent with our findings using the hot-plate assay, while the antiallodynic effects of oxycodone are maintained in RGS9WT mice throughout the monitoring period, RGS9KO mice show decreased analgesic response to oxycodone by day 12 (Figure 5c).

\section{DISCUSSION}

Our findings provide new information on the intracellular pathways involved in behavioral responses to oxycodone, in pain-free as well as in neuropathic pain states. RGS9-2 is a key modulator of drug addiction, and has been shown to have a dynamic role in the actions of several drug of abuse, including cocaine, amphetamine, and morphine (Traynor et al, 2009). Our data reveal that although oxycodone has rewarding, locomotor activating, and analgesic effects that are similar to morphine, RGS9-2 has distinct modulatory roles in the actions of these two MOR-targeting opioids. RGS9-2 promotes the rewarding and locomotor sensitizing effects of oxycodone, and opposes the development of analgesic tolerance. This is the opposite of what we observed in previous studies using morphine, where we found that RGS9-2 opposes the development of morphine reward, and promotes the development of analgesic tolerance (Zachariou et al, 2003; Gaspari et al, 2014). In addition, although deletion of the $R g s 9$ gene exacerbates many morphine withdrawal symptoms (Zachariou et al, 2003), it does not affect symptoms of oxycodone withdrawal. Thus, RGS9-2 appears to uniquely modulate morphine, as analgesic responses to oxycodone, fentanyl, and methadone are positively modulated by RGS9-2 (Psifogeorgou et al, 2011). The distinct phenotypes observed with morphine vs oxycodone may lie in different intracellular mechanisms by which these compounds exert their effects; for example, recruitment of different $\mathrm{G} \alpha$ subunits (Psifogeorgou et al, 2011), or different effectors activated by each drug, although differences owing to receptor specificity or receptor dimer formation should also be considered. We speculate that similar to fentanyl and methadone, oxycodone in the striatum promotes short-term complexes between RGS9-2, MOR, and $\mathrm{G} \alpha_{\mathrm{q}}$ subunits, whereas morphine promotes stable complexes between RGS9-2-G $\beta 5$, MOR, and $\mathrm{G} \alpha_{\mathrm{i}}$ subunits (Psifogeorgou et al, 2011). Therefore, genetic inactivation of Rgs9 will differentially affect cellular and behavioral responses to oxycodone and morphine.

RGS9-2 is very abundant in the striatum, but it may also affect MOR function in the spinal cord (Terzi et al, 2009) and other supraspinal sites expressing MOR, including the periaqueductal gray (PAG) (Garzón et al, 2005), Moreover, the actions of MOR may be mediated in a cell type- or region-specific level by other members of the RGS family, including RGS4 and RGSz proteins (Gold et al, 2003; Garzón et al, 2005).

A large number of in vitro and in vivo studies have provided information on the mechanisms underlying the acute and chronic actions of MOR agonists (Muller and Unterwald 2004; Koch et al, 2005; Raehal and Bohn, 2005; Pradhan et al, 2006; Walwyn et al, 2010; Quillinan et al, 2011). The majority of these studies have focused on morphine and heroin, and have provided insight into signal-transduction events associated with addiction or the development of analgesic tolerance. Although oxycodone is highly prescribed for the treatment of acute and chronic pain conditions, there is very limited information on the cellular events triggered by repeated oxycodone administration, and the G-protein complexes that modulate oxycodone actions in specific brain regions or cellular populations modulating addiction and analgesia. Several recent reports have used models of reward and drug self-administration to study the actions of oxycodone in the adolescent and adult life 
a

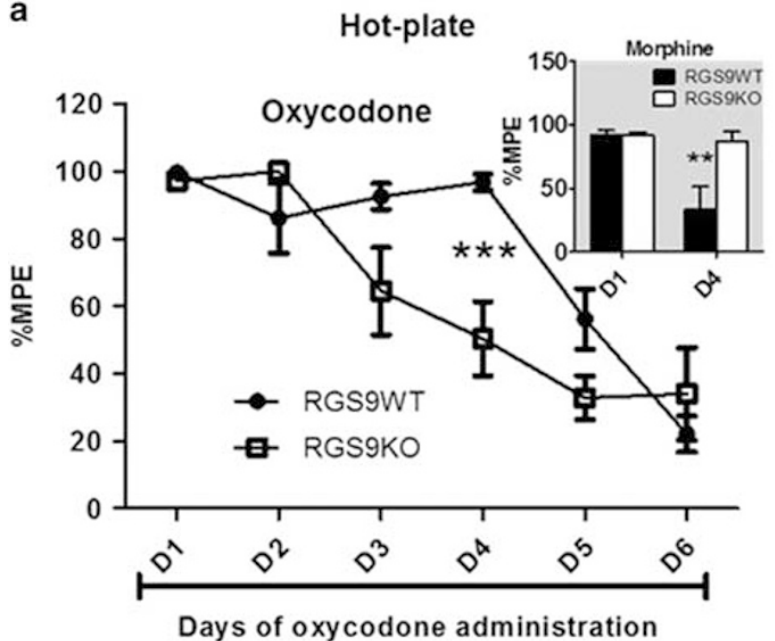

b
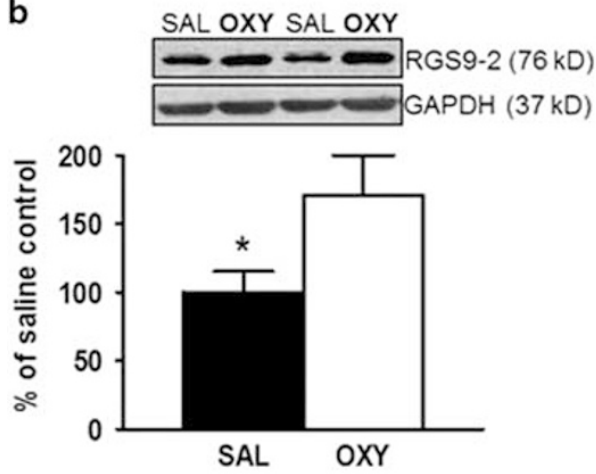

C

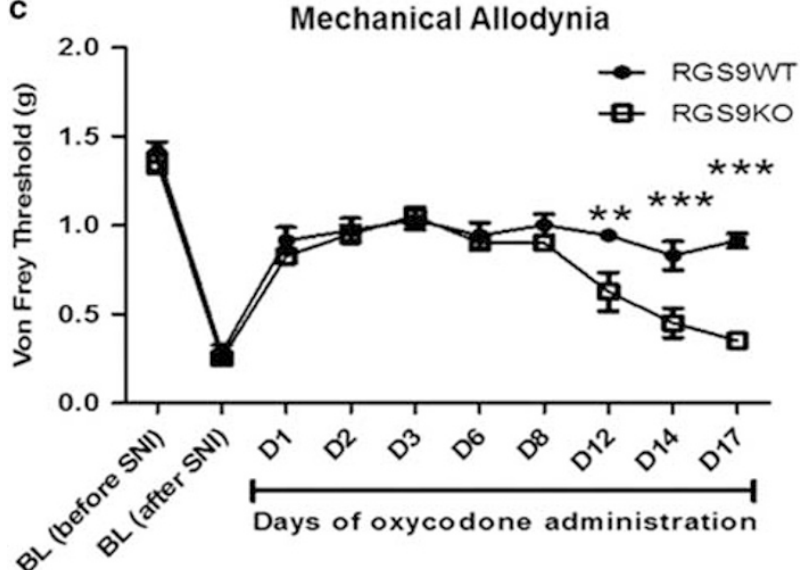

Figure 5 Regulator of G-protein signaling 9-2 (RGS9-2) acts as a negative modulator of oxycodone tolerance. (a) RGS9-knockout (KO) mice become tolerant to the analgesic effects of the drug earlier than their wild-type controls (dose: $20 \mathrm{mg} / \mathrm{kg}$, two-way analysis of variance (ANOVA) repeatedmeasures followed by Bonferroni post hoc test, $F(I, 45)=3.77$, **** $<0.00$ I). Upper panel: The opposite phenotype is observed when morphine is used in the hot-plate assay, as RGS9KO mice respond to morphine at a time point their wild-type controls show analgesic tolerance (dose: $20 \mathrm{mg} / \mathrm{kg}$, two-way ANOVA repeated-measures followed by Bonferroni post hoc test, $F(I, 7)=9.25$, $* * * 0.0 \mathrm{I}$ ). (b) Furthermore, protein levels of RGS9-2 are upregulated in the nucleus accumbens (NAc) of mice tolerant to oxycodone ( $t$-test, $t(28)=2.14, * p<0.05)$. (c) RGS9KO mice experiencing neuropathic pain become tolerant to the antiallodynic effects of the drug earlier than their wild-type controls. Oxycodone treatment was initiated on day I 5 after spared nerve injury (SNI) surgery, when mechanical allodynia levels were identical between genotypes. The drug was administered once a day for 17 consecutive days, and Von Frey responses were monitored I h after drug injection (dose: $3 \mathrm{mg} / \mathrm{kg}$, two-way ANOVA repeated-measures followed by Bonferroni post hoc tests, $F(I, I \mid 7)=10.49$, *** $p<0.0$ I, **** $p<0.00$ I).

(Emery et al, 2015). Further understanding of the longterm adaptation oxycodone treatment promotes in the reward pathway, and the exact signal-transduction events triggered by oxycodone, will help design better treatment strategies for pain management and identify factors that contribute to oxycodone abuse vulnerability.

Chronic pain-induced plasticity in addiction- and analgesia-related brain networks may affect sensitivity to opiate analgesics (Navratilova and Porreca, 2014; Taylor et al., 2015; Mitsi and Zachariou, 2016). Our studies examined the role of the striatal-enriched RGS9-2 in oxycodone actions under neuropathic pain states. Our findings from mice tested at 2 weeks after SNI reveal that neuropathic pain states reduce oxycodone reward sensitivity in both RGS9WT and RGS9KO animals, consistent with the hypothesis that chronic pain states impact the brain reward network (Mitsi and Zachariou, 2016). Decreased reward sensitivity under chronic pain states has been reported by several other groups (Ozaki et al, 2002, 2003; Ewan and Martin, 2011; Navratilova et al, 2012; Wu et al, 2014), but this is the first study to examine oxycodone reward in the context of chronic pain. Notably, reports also show that longterm inflammatory pain may promote heroin selfadministration (Hipolito et al, 2015) and therefore it is important to assess the impact of inflammatory and neuropathic pain in oxycodone self-administration and other addiction-related paradigms. Future studies should monitor reward sensitivity to several pain killers at later time points after nerve injury, when depression symptoms are also present, to understand how long-term pain states affect the function of the reward pathway. In addition to G-protein complexes, neuroimmune and other adaptations affect the function of MOR and other receptors in the brain reward center under chronic pain states, and contribute to the decreased reward sensitivity observed in the CPP paradigm. For example, recent studies showed that in the rat PAG, MOR may function differently in pain-free $v s$ chronic pain states (Eidson and Murphy, 2013; Mehalick et al, 2013) and have demonstrated the potent role of Toll-like receptors in modulating morphine tolerance in models of acute and longterm inflammatory pain. 
Our data from SNI groups of mice also support a protective modulatory role of RGS9-2 in oxycodone tolerance: in accord with our findings from experiments using the hot-plate assay, $\mathrm{KO}$ of the Rgs9 gene does not affect the acute action of oxycodone on mechanical allodynia following SNI. However, when the drug is administered for consecutive days, the antiallodynic response is abolished in RGS9KO mice after 12 days of treatment, reflecting the development of tolerance. Therefore, RGS9-2 complexes protect against the development of analgesic tolerance to oxycodone in models of acute and chronic pain.

RGS9-2 has a prominent role in the modulation of GPCR responses in the striatum, where it is expressed in very high levels, but it is absent or expressed in low amounts in other brain regions expressing MORs (Traynor et al, 2009). We suggest that the phenotypes observed are due to loss of RGS9-2 function primarily in the striatum, and that other members of the RGS family, including RGS7, RGS4, and RGS20 may control MOR function in other brain regions associated with addiction or analgesia, including the locus coeruleus and the PAG (Han et al, 2010; Gold et al, 2003; Garzón et al, 2005).

Future work will investigate the signal-transduction and gene expression changes induced by oxycodone exposure in the brain reward center, and the cellular and molecular determinants of oxycodone addiction. While the present study focuses on groups of male mice, future work will further determine the role of RGS9-2 in behavioral responses to oxycodone and other pain killers in male and female animals. Our findings that RGS9-2 modulates the actions of oxycodone in a manner distinct from its modulation of morphine support the notion that although synthetic opioid analgesics produce similar physiological responses, they act via distinct intracellular mechanisms. This knowledge may lead to the development of more efficacious and less addictive compounds and will help developing better strategies for the management of chronic pain.

\section{FUNDING AND DISCLOSURE}

The authors declare no conflict of interest.

\section{ACKNOWLEDGMENTS}

This work was supported by the Greek Secretariat of Research and Technology-Greek Ministry of Education and Religious Sffairs, the 7th EU Framework (Aristia I) and by NINDS (NS086444).

\section{REFERENCES}

Anderson GR, Semenov A, Song JH, Martemyanov KA (2007). The membrane anchor R7BP controls the proteolytic stability of the striatal specific RGS protein, RGS9-2. J Biol Chem 282: 4772-4781.

Bailey CP, Connor M (2005). Opioids: cellular mechanisms of tolerance and physical dependence. Curr Opin Pharmacol 5: 60-68.

Baliki MN, Petre B, Torbey S, Herrmann KM, Huang L, Schnitzer TJ et al (2012). Corticostriatal functional connectivity predicts transition to chronic back pain. Nat Neurosci 15: 1117-1119.
Baliki MN, Apkarian AV (2015). Nociception, pain, negative moods, and behavior selection. Neuron 87: 474-491.

Butler SF, Black RA, Cassidy TA, Dailey TM, Budman SH (2011). Abuse risks and routes of administration of different prescription opioid compounds and formulations. Harm Reduct J 8: 29.

Carise D, Dugosh KL, Mclellan AT, Camilleri A, Woody GE, Lynch KG (2007). Prescription OxyContin abuse among patients entering addiction treatment. Am J Psychiatry 164: 1750-1756.

Charlton JJ, Allen BP, Psifogeorgou K, Chakravarty S, Gomes I, Neve RL et al (2008). Multiple actions of spinophilin modulate mu opioid receptor function. Neuron 58: 238-247.

Collins D, Reed B, Zhang Y, Kreek MJ (2016). Sex differences in responsiveness to the prescription opioid oxycodone in mice. Pharmacol Biochem Behav 148: 99-105.

Comer SD, Sullivan MA, Whittington RA, Vosburg SK, Kowalczyk WJ (2007). Abuse liability of prescription opioids compared to heroin in morphine-maintained heroin abusers. Neuropsychopharmacology 33: 1179-1191.

Dart RC, Surratt HL, Cicero TJ, Parrino MW, Severtson SG, Bucher-Bartelson B et al (2015). Trends in opioid analgesic abuse and mortality in the United States. $N$ Engl J Med 372: 1573-1574.

Eidson LN, Murphy AZ (2013). Blockade of Toll-like receptor 4 attenuates morphine tolerance and facilitates the pain relieving properties of morphine. J Neurosci 33: 15952-15963.

Emery MA, Bates MS, Wellman PJ, Eitan S (2015). Differential effects of oxycodone, hydrocodone, and morphine on the responses of D2/D3 dopamine receptors. Behav Brain Res 284: 37-41.

Ewan EE, Martin TJ (2011). Opioid facilitation of rewarding electrical brain stimulation is suppressed in rats with neuropathic pain. Anesthesiology 14: 624-632.

Garzón J, Rodríguez-Muñoz M, Sánchez-Blázquez P (2005). Morphine alters the selective association between mu-opioid receptors and specific RGS proteins in mouse periaqueductal gray matter. Neuropharmacology 48: 853-868.

Gaspari S, Papachatzaki MM, Koo JW, Carr FB, Tsimpanouli ME, Stergiou E et al (2014). Nucleus accumbens-specific interventions in RGS9-2 activity modulate responses to morphine. Neuropsychopharmacology 39: 1968-1977.

Gold SJ, Han MH, Herman AE, Ni YG, Pudiak CM, Aghajanian GK et al (2003). Regulation of RGS proteins by chronic morphine in rat locus coeruleus. Eur J Neurosci 17: 971-980.

Gruccu G (2007). Treatment of painful neuropathy. Curr Opin Neurol 20: 531-535.

Han $\mathrm{MH}$, Renthal W, Ring RH, Rahman Z, Psifogeorgou K, Howland D et al (2010). Brain region specific actions of regulator of $\mathrm{G}$ protein signaling 4 oppose morphine reward and dependence but promote analgesia. Biol Psychiatry 67: 761-769.

Hermanns K, Junker U, Nolte T (2012). Prolonged-release oxycodone/naloxone in the treatment of neuropathic painresults from a large observational study. Expert Opin Pharmacother 13: 299-311.

Hipolito L, Wilson-Poe A, Campos-Jurado Y, Zhong E, Gonzalez-Romero J, Virag L et al (2015). Inflammatory pain promotes increased opioid self-administration: role of dysregulated ventral tegmental area opioid receptors. J Neurosci 35: 12217-12231.

Hollinger S, Hepler J (2002). Cellular regulation of RGS proteins: modulators and integrators of $\mathrm{G}$ protein signaling. Pharmacol Rev 54: 527-559.

Jayaraman M, Zhou H, Jia L, Cain MD, Blumer KJ (2009). R9AP and R7BP: traffic cops for the RGS7 family in phototransduction and neuronal GPCR signaling. Trends Pharmacol Sci 30: 17-24.

Kimple AJ, Bosch DE, Giguere PM, Siderovski DP (2011). Regulators of G-protein signaling and their substrates: promises and challenges in their use as drug discovery targets. Pharmacol Rev 63: 728-749. 
Koch T, Widera A, Bartzsch K, Schulz S, Brandenburg LO, Wundrack $\mathrm{N}$ et al (2005). Receptor endocytosis counteracts the development of opioid tolerance. Mol Pharmacol 67: 280-287.

Kovoor A, Seyffarth P, Ebert J, Barghshoon S, Chen CK, Schwarz S et al (2005). D2 dopamine receptors colocalize regulator of G-protein signaling 9-2 (RGS9-2) via the RGS9 DEP domain, and RGS9 knock-out mice develop dyskinesias associated with dopamine pathways. J Neurosci 25: 2157-2165.

Ling W, Mooney L, Hillhouse M (2011). Prescription opioid abuse, pain and addiction: clinical issues and implications. Drug Alcohol Rev 30: 300-305.

Mehalick ML, Ingram SL, Aicher SA, Morgan MM (2013). Chronic Inflammatory pain prevents tolerance to the antinociceptive effect of morphine microinjected into the ventrolateral periaqueductal gray of the rat. J Pain 12: 1601-1610.

Mitsi V, Terzi D, Purushothaman I, Manouras L, Gaspari S, Neve RL et al (2015). RGS9-2-controlled adaptations in the striatum determine the onset of action and efficacy of antidepressants in neuropathic pain states. Proc Natl Acad Sci USA 112: E5088-E5097.

Mitsi V, Zachariou V (2016). Modulation of pain, nociception, and analgesia by the brain reward center. Neuroscience 338: 81-92.

Muller DL, Unterwald EM (2004). In vivo regulation of extracellular signal-regulated protein kinase (ERK) and protein kinase B (Akt) phosphorylation by acute and chronic morphine. J Pharmacol Exp Ther 310: 774-782.

Navratilova E, Xie JY, Okun A, Qu C, Eyde N, Ci S et al (2012). Pain relief produces negative reinforcement through activation of mesolimbic reward-valuation circuitry. Proc Natl Acad Sci USA 109: 20709-20713.

Navratilova E, Porreca F (2014). Reward and motivation in pain and pain relief. Nat Neurosci 17: 1304-1312.

Niikura K, Zhang Y, Ho A, Kreek M (2013). Oxycodone-induced conditioned place preference and sensitization of locomotor activity in adolescent and adult mice. Pharmacol Biochem Behav 110: 112-116.

Ozaki S, Narita M, Narita M, Iino M, Sugita J, Matsumura Y et al (2002). Suppression of the morphine-induced rewarding effect in the rat with neuropathic pain: implication of the reduction in mu-opioid receptor functions in the ventral tegmental area. J Neurochem 82: 1192-1198.

Ozaki S, Narita M, Narita M, Iino M, Miyoshi K, Suzuki T (2003). Suppression of the morphine-induced rewarding effect and G-protein activation in the lower midbrain following nerve injury in the mouse: involvement of G-protein-coupled receptor kinase 2. Neuroscience 116: 89-97.

Pradhan A, Siau C, Constantin A, Clarke P (2006). Chronic morphine administration results in tolerance to delta opioid receptor-mediated antinociception. Neuroscience 141: 947-954.

Psifogeorgou K, Papakosta P, Russo SJ, Neve RL, Kardassis D, Gold SJ et al (2007). RGS9-2 is a negative modulator of $\mu$-opioid receptor function. J Neurochem 103: 617-625.

Psifogeorgou K, Terzi D, Papachatzaki MM, Varidaki A, Ferguson D, Gold SJ et al (2011). A unique role of RGS9-2 in the striatum as a positive or negative regulator of opiate analgesia. J Neurosci 31: 5617-5624.

Quillinan N, Lau EK, Virk M, Zastrow MV, Williams JT (2011). Recovery from $\mu$-opioid receptor desensitization after chronic treatment with morphine and methadone. J Neurosci 31: 4434-4443.

Raehal KM, Bohn LM (2005). Mu opioid receptor regulation and opiate responsiveness. AAPS J 7: E587-E591.

Rahman Z, Schwarz J, Gold SJ, Zachariou V, Wein MN, Choi K-H et al (2003). RGS9 modulates dopamine signaling in the basal ganglia. Neuron 38: 941-952.
Ray WA, Chung CP, Murray KT, Hall K, Stein CM (2016). Prescription of long-acting opioids and mortality in patients with chronic noncancer pain. JAMA 315: 2415-2423.

Sanchez V, Carpenter MD, Yohn NL, Blendy JA (2016). Longlasting effects of adolescent oxycodone exposure on rewardrelated behavior and gene expression in mice. Psychopharmacology 233: 3991-4002.

Schwartz N, Temkin P, Jurado S, Lim BK, Heifets BD, Polepalli JS et al (2014). Decreased motivation during chronic pain requires long-term depression in the nucleus accumbens. Science 345: 535-542.

Shields SD, Eckert WA, Basbaum AI (2003). Spared nerve injury model of neuropathic pain in the mouse: a behavioral and anatomic analysis. J Pain 4: 465-470.

Spiller H, Lorenz DJ, Bailey EJ, Dart RC (2009). Epidemiological trends in abuse and misuse of prescription opioids. J Addict Dis 28: $130-136$.

Stratinaki M, Varidaki A, Mitsi V, Ghose S, Magida J, Dias C et al (2013). Regulator of G protein signaling 4 is a crucial modulator of antidepressant drug action in depression and neuropathic pain models. Proc Natl Acad Sci USA 110: 8254-8259.

Taylor AMW, Castonguay A, Taylor AJ, Murphy NP, Ghogha A, Cook C et al (2015). Microglia disrupt mesolimbic reward circuitry in chronic pain. J Neurosci 35: 8442-8450.

Terzi D, Stergiou E, King SL, Zachariou V (2009). Regulators of G protein signaling in neuropsychiatric disorders. Prog $\mathrm{Mol}$ Biol Transl Sci 86: 299-333.

Terzi D, Cao Y, Agrimaki I, Martemyanov KA, Zachariou V (2011). R7BP modulates opiate analgesia and tolerance but not withdrawal. Neuropsychopharmacology 37: 1005-1012.

Terzi D, Gaspari S, Manouras L, Descalzi G, Mitsi V, Zachariou V (2014). RGS9-2 modulates sensory and mood related symptoms of neuropathic pain. Neurobiol Learn Mem 115: 43-48.

Traynor JR, Terzi D, Caldarone BJ, Zachariou V (2009). RGS9-2: probing an intracellular modulator of behavior as a drug target. Trends Pharmacol Sci 30: 105-111.

Walwyn WM, Miotto KA, Evans CJ (2010). Opioid pharmaceuticals and addiction: The issues, and research directions seeking solutions. Drug Alcohol Depend 108: 156-165.

Wu Y, Na X, Zang Y, Cui Y, Xin W, Pang R et al (2014). Upregulation of tumor necrosis factor-alpha in nucleus accumbens attenuates morphine-induced rewarding in a neuropathic pain model. Biochem Biophys Res Commun 449: 502-507.

Yalcin I, Barthas F, Barrot M (2014). Emotional consequences of neuropathic pain: Insight from preclinical studies. Neurosci Biobehav Rev 47: 154-164.

Zachariou V, Georgescu D, Sanchez N, Rahman Z, Dileone R, Berton O et al (2003). Essential role for RGS9 in opiate action. Proc Natl Acad Sci USA 100: 13656-13661.

Zhang Y, Mayer-Blackwell B, Schlussman SD, Randesi M, Butelman ER, Ho A et al (2013). Extended access oxycodone self-administration and neurotransmitter receptor gene expression in the dorsal striatum of adult C57BL/6J mice. Psychopharmacology 231: 1277-1287.

Zhang Y, Brownstein A, Buonora M, Niikura K, Ho A, Rosa JCD et al (2015). Self administration of oxycodone alters synaptic plasticity gene expression in the hippocampus differentially in male adolescent and adult mice. Neuroscience 285: 34-46.

Zhang Y, Windisch K, Altschuler J, Rahm S, Butelman ER, Kreek MJ (2016). Adolescent oxycodone self administration alters subsequent oxycodone-induced conditioned place preference and anti-nociceptive effect in C57BL/6J mice in adulthood. Neuropharmacology 111: 314-322.

Zhu Y, Zhang H (2015). Evidence for the contribution of genetic variations in regulator of $G$ protein signaling 9 to the genetic susceptibility of heroin dependence. Mol Med Rep 11: 3908-3913. 